\title{
A ZERO-ONE LAW FOR GAUSSIAN PROCESSES
}

\author{
NARESH C. JAIN ${ }^{1}$
}

Abstract. Let $P_{0}$ be a Gaussian probability measure on the measurable space $(X, B(X))$, where $X$ is a linear space of realvalued functions over a complete separable metric space $T$, and $B(X)$ is the $\sigma$-algebra generated by sets of the form $\left\{x \in X:\left(x\left(t_{1}\right)\right.\right.$, $\left.\left.\cdots, x\left(t_{n}\right)\right) \in B^{n}\right\} ; B^{n}$ being the Borel sets of $R^{n}, n \geqq 1$. The covariance $R(s, t)$ is assumed continuous on $T \times T$. If $G$ is a subgroup of $X$ and belongs to the $\sigma$-algebra $B_{0}(X)$ (the completion of $B(X)$ with respect to $P_{0}$ ), then it is shown that $P_{0}(G)=0$ or 1 .

1. Introduction. For the background and history of the problem we refer to [1] and [2]. We would like to point out here that Jamison and Orey in [1] proved the above result for the special case where the Gaussian process involved had continuous paths. Kallianpur [2] proved such a result for $r$-modules (groups closed under multiplication by rationals). Kallianpur's result for groups is restricted to those which are $B(X)$-measurable rather than $B_{0}(X)$-measurable. He points out in [2] why his proof does not work for $B_{0}(X)$-measurable subgroups. Our main result (Theorem 1 ) unifies and generalizes the results of [1] and [2], and also gives an answer in the affirmative to the conjecture made in [1]. Our method of proof is similar to the one given in [2], but it is simpler. The notation used here is also essentially the same as in [2].

$T$ is a complete separable metric space. $X$ is a linear space of realvalued functions defined on $T$ with the usual operation of addition of functions and multiplication by real scalars. $B(X)$ is the $\sigma$-algebra as explained above. $P_{0}$ is a Gaussian measure on $(X, B(X))$. We assume that

$$
\begin{gathered}
\int_{X} x(t) P_{0}(d x)=0 \quad \text { for each } t \in T ; \\
\int_{X} x(s) x(t) P_{0}(d x)=R(s, t), \quad s, t \in T,
\end{gathered}
$$

is continuous on $T \times T . H(R)$ will denote the reproducing kernel Hilbert space of the covariance $R$. It is also assumed that $H(R) \subset X$.

Received by the editors November 18, 1970.

A MS 1970 subject classifications. Primary 60G15.

Key words and phrases. Gaussian process, zero-one law

1 This research was partially supported by NSF. 
$B_{0}(X)$ denotes the completion of the $\sigma$-algebra $B(X)$ with respect to $P_{0}$.

The main result is the following:

TheOREM 1. Let $G$ be a $B_{0}(X)$-measurable subgroup of the linear space $X$. Then $P_{0}(G)=0$ or 1 .

REMARK. For applications of this result we refer to Lemma 5 [2].

2. Proof of Theorem 1. We need the following lemmas.

LEMmA 1. Let $F \in B_{0}(X)$. If $m \in H(R)$ and $\alpha$ is a real number, then the set

$$
F_{\alpha}=\{x \in X: x=y+\alpha m, y \in F\}
$$

is in $B_{0}(X)$, and

$$
\lim _{\alpha \rightarrow 0} P_{0}\left(F_{\alpha}\right)=P_{0}(F) .
$$

Proof. This is established in the proof of Lemma 5 [2].

LEMMA 2. Let $\left\{e_{j}\right\}_{1}^{\infty}$ be a complete orthonormal system in $H(R)$ and $g$ be a $B_{0}(X)$-measurable real function such that for each $x \in X$ and every rational $r g\left(x+r e_{j}\right)=g(x), j=1,2, \cdots$. Then $g(x)=$ constant a.s. $\left(P_{0}\right)$.

Proof. See Lemma 6 [2].

To finish the proof of the theorem, let $P_{0}(G)>0$. We will show that this implies that $H(R) \subset G$. It is then seen easily that $P_{0}(G)=1$ as follows: since $G$ is a group and $H(R)$ a Hilbert space with a complete orthonormal system $\left\{e_{j}\right\}$, it follows that $x \in G$ if and only if $x+r e_{j} \in G$ for every rational $r, j=1,2, \ldots$. Let $I_{G}$ be the indicator function of the set $G$, then by Lemma 2 we have $I_{G}=$ constant a.s. $\left(P_{0}\right)$. But this constant must be equal to 1 since $P_{0}(G)>0$.

It thus remains to show that $P_{0}(G)>0$ implies that $H(R) \subset G$. There exists a positive integer $s$ such that $P_{0}(G)>1 / s$. We hold this $s$ fixed. Let $m \in H(R), m \notin G$. This will lead to a contradiction. For each positive integer $n$ define $s+1$ sets $G_{0}^{(n)}, G_{1}^{(n)}, \cdots, G_{s}^{(n)}$ as follows:

$$
\left.G_{0}^{(n)}=G, \quad G_{k}^{(n)}=\left\{x: x=y+(s ! k n)^{-1} m, y \in G\right\}, 1 \leqq k \leqq s\right\} .
$$

By Lemma 1 these sets are in $B_{0}(X)$ and

$$
\lim _{n} P_{0}\left(G_{k}^{(n)}\right)=P_{0}(G), \quad 0 \leqq k \leqq s .
$$

We now show that these sets must be pairwise disjoint. If $G_{0}^{(n)}$ and 
$G_{\mathbf{k}}^{(n)}, k>0$, have an element in common, then for some $x, y \in G$, $x=y+(s ! k n)^{-1} m$; hence $m=(s ! k n)(x-y)$, an element of $G$, which contradicts that $m \notin G$. Suppose now that $G_{j}^{(n)}, G_{\boldsymbol{z}}^{(n)}, 1 \leqq j<k$, have an element in common. Then for some $x, y \in G$,

$$
x+(s ! j n)^{-1} m=y+(s ! k n)^{-1} m .
$$

Hence $m=(s ! k j n)(k-j)^{-1}(y-x)$. But $(s ! k j n)(k-j)^{-1}$ is a positive integer and this implies again that $m \in G$, a contradiction. We thus have $\sum_{k=0}^{s} P_{0}\left(G_{k}^{(n)}\right) \leqq 1, n=1,2, \cdots$. Letting $n$ tend to infinity, we conclude from $(2.3)$ that $(s+1) P_{0}(G) \leqq 1$. Since $P_{0}(G)>1 / s$, this is a contradiction. Hence $H(R) \subset G$ and the proof is complete.

\section{REFERENCES}

1. B. Jamison and S. Orey, Subgroups of sequences and paths, Proc. Amer. Math. Soc. 24 (1970), 739-744. MR 40 \#8121.

2. G. Kallianpur, Zero-one laws for Gaussian processes, Trans. Amer. Math. Soc. 149 (1970), 199-211.

University of Minnesota, Minneapolis, Minnesota 55455 